\title{
De Novo Classification Request
}

National Cancer Institute

\section{Source}

National Cancer Institute. De Novo Classification Request. NCI Thesaurus. Code C121843.

A premarket submission made to the FDA for classification of a device for which general controls or general and special controls provide a reasonable assurance of safety and effectiveness, but for which there is no legally marketed predicate device. 